\title{
Impact of environment color on individual responses in public spaces of shopping malls
}

\author{
Fashu Yi ${ }^{\mathrm{a}}$, Jian Kang ${ }^{\mathrm{a}, \mathrm{b}, *}$ \\ a School of Architecture, Harbin Institute of Technology, Key Laboratory of Cold \\ Region Urban and Rural Human Settlement Environment Science and \\ Technology, Ministry of Industry and Information Technology, Harbin 150001, \\ China \\ ${ }^{b}$ UCL Institute for Environmental Design and Engineering, The Bartlett, \\ University College London (UCL), London WC1H ONN, United Kingdom \\ * Corresponding author
}

\begin{abstract}
This study aims to explore the specific impact of a color's hue, saturation, and brightness value on people's evaluation, behavior, and emotions in the public space of existing shopping malls. Following a field investigation, color composition and distribution characteristics in the public spaces of shopping malls are summarized. In the real scenes, the hues of colors are mainly warm colors, with some supplemented by blue. The saturation of the majority colors, which are almost greyish colors, is lower than $33 \%$ and their brightness is very wide and evenly distributed. An experiment was then conducted in the laboratory, wherein photos taken and sounds recorded on-site were shown to participants; the participants were then asked to answer questionnaires based on the pleasure-arousal-dominance emotion and approach-avoidance behavior theories. The results showed that hue has a weak effect on individual responses, whereas saturation was found to have a significant effect. Moreover, the brightness difference between colors can cause a change in satisfaction, behavior intention, and emotion. Additionally, in the public spaces of shopping malls, the red color is more associated with embodied meaning, whereas the green color is more associated with referential meaning, in which different objects have significantly different influences.
\end{abstract}

Keywords: color, HSB, individual responses, public space, shopping malls

Color Research and Application

Date Received: 5 June 2019 Date Accepted: 8 January 2020 


\section{Introduction}

People act differently, with regard to their emotions and behavior, according to the surrounding context and environmental stimuli. Many studies have been conducted and several theory models created to help explain the responses to external stimuli. In particular, color is an attribute that cannot be ignored in the visual environment. Many researchers have studied the influence of color's various attributes on different aspects, such as the effect of a color's hue, saturation, design, size, arrangement, or environmental lighting, on a human's attitudes evaluation, behavior intention, emotion, brand recognition, and so on. In such scenarios, typical carriers of color include room walls, product packaging, or advertising [1].

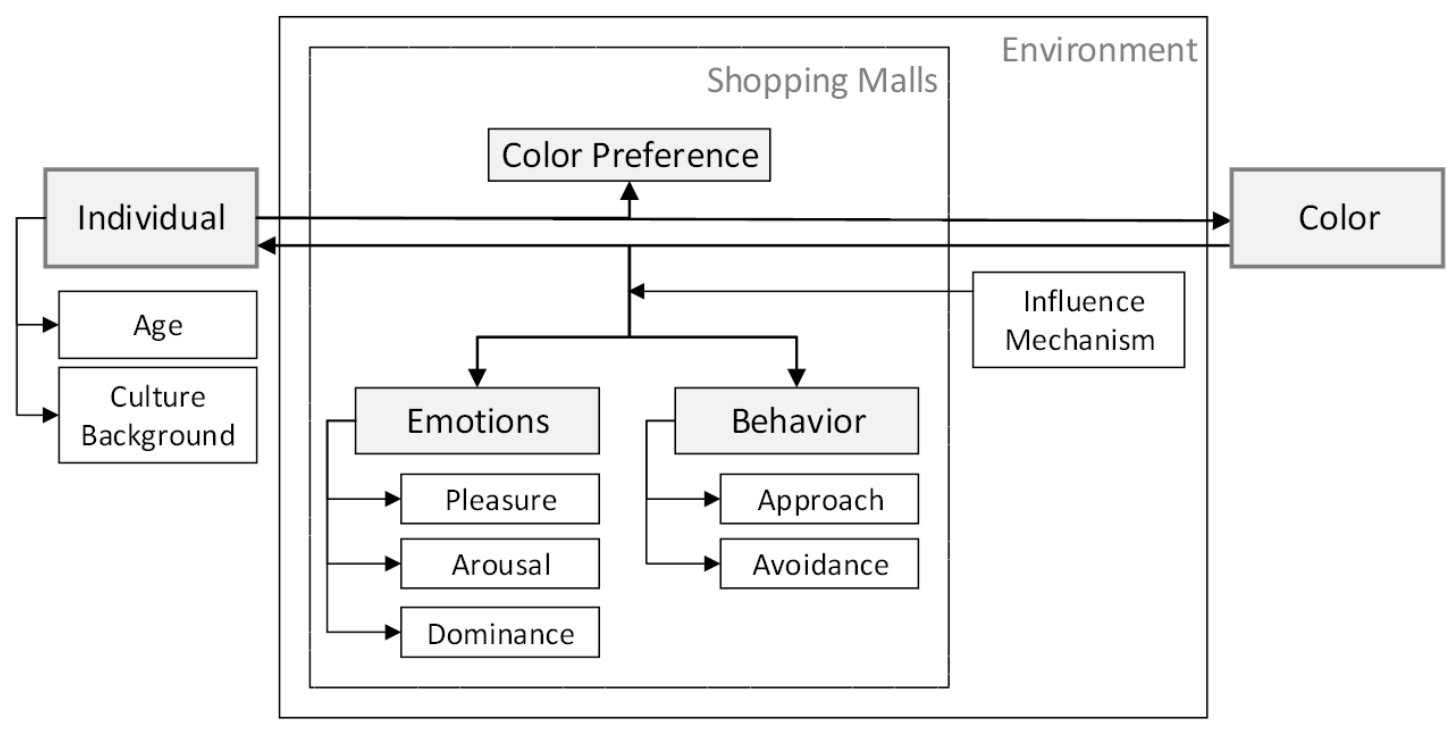

Fig. 1. Outline of Literature Review.

Among the literature regarding color research, studies on color preference were conducted first. Guilford and Smith [2] conducted a systematic study on color preference in 1959. A sequence of hue preference was arranged, and it was found that brighter and more saturated colors were considered more pleasant. Subsequently, it was found that color preference was influenced by color wavelengths [3], color coordination [4], environment color and object color [5], and a prediction model of color preference was developed [4].

Research in color psychology [6,7] shows that color itself has meaning, and specific color clues can affect emotion and behavior. Based on the Pleasure-Arousal-Dominance (PAD) emotion measurement method, it is inferred that hues of blue, blue-green, green, redpurple, purple, and purple-blue are the most pleasant colors, whereas yellow and yellowgreen are the most unpleasant colors. Meanwhile green-yellow, blue-green, and green are the most energetic, while violet-blue and yellow-red are the least awakened. Compared to red-purple, green-yellow can significantly improve the sense of dominance [8]. Brightness is also one of the important sources of perceptual information because the difference in brightness provides contrast, which is essential for vision [9]. Brighter images receive a more positive evaluation, whereas darker images obtain a more 
negative evaluation [10]. For behavior, it was observed that red leads to avoidance behavior but can increase attention and memory, whereas blue can activate approach behavior [11].

While studying color preferences and their influence on people in general, researchers began to pay attention to the differences among the color preferences in specific fields. For example, architects and interior designers pay more attention to the influence of colors in the building environment, and managers focus on the effect of color on shopping atmosphere and behavior. Kotler [12] introduced the concept of store atmospherics in 1973, pointing out that a commercial atmosphere can influence consumer behavior by attention, information communication, and emotion. Color is an important element of a commercial environment. From the perspective of evaluation, as the color wavelength changes from orange-red to blue, a better evaluation, higher patronage, and purchase intention can be obtained, including a stimulation of shopping, less purchase delay, and higher tendencies of shopping and browsing $[3,13,14]$. Adding bright or soft light in the interior will change the evaluation result as compared to that with no light [15]. As for the arousal aspect, red and blue are beneficial in improving arousal and, thus, trigger purchase behavior, whereas green has a low arousal level and is suitable for the waiting area [3]. Cold colors create a more relaxed shopping environment, whereas warm colors make people more willing to be close to the environment [15].

The influence of color on individuals is also different among different groups. Sevenyear-old children like yellow and red the most, whereas adults prefer blue, red, and green but do not like white, red, and black. Adults within the age range of 20-56 years do not like yellow, and the preference for green increases with age [16]. Additionally, some studies have focused on people from different cultural backgrounds and found that there were significant differences in the cognition of commodity quality between FrenchCanadians and Anglo-Canadians under the same color environment [17].

Many researchers have also attempted to explain the effect of color on individual responses on the basis of physiology, psychophysics, emotional evoking, ecological valence theory, and other aspects; e.g., such studies include: physiological studies on the characteristics of the visual receptors' sensory stimuli, [18] investigations of psychophysical stimuli that cause sensation, $[19,20]$ consideration of the stimulation by emotional colors for specific emotions [4], and investigations of the consistency between color preferences in ecological valence theory and those in common objects with corresponding colors [19,20]. Furthermore, many studies have confirmed that there are two different aspects to the meaning of color, as with music. One aspect is the embodied meaning, which results from attributes embodied in the aesthetic stimulus independent of the context and semantic content [1,21]. For example, long wavelength colors, such as red, can implement arousal to a higher level of stimulation. The other aspect is the referential meaning, generated by the semantic association derived from the aesthetic stimulus, which is acquired from and depends on the clues of the surrounding environment [22]; therefore, it may vary from culture to culture.

However, in previous studies related to color (Figure 1), there have been few analyses on color characteristics in a real environment as the independent variables. Such an analysis typically involves two main parts: 1 ) assigning a specific color to a certain object and exploring individual color preferences; 2 ) decorating a laboratory environment with 
specific colors and studying the influence of colors on individual responses. In this kind of research, colors that should be regarded as the environment are arranged in the foreground, which are observed, perceived, and evaluated like a single object of one or several specific colors. However, the reality is that people perform activities in a comprehensive environment composed of a variety of colors, and scenes with single or certain pure colors are very rare. On the one hand, the perceived difference with a real environment may cause discomfort for the participants; on the other hand, the difference in the perception mechanism between gazing at the foreground object and glancing at the background environment may influence the experimental results. Additionally, for the dependent variable, most previous studies have aimed at improving store profitability. The purpose of studying emotion and behavior has been to improve the indices that are beneficial to business indicators, such as staying time, impulse buying opportunities, and turnover frequency. Moreover, the space which is the focus of attention is usually a space whose primary function is the sale of goods and presentation of display items, but rarely public spaces for walking and relaxing.

Therefore, the aim of this study is to investigate the impact of environment color on individual responses. Individual responses refer to the individual's satisfaction, "approach-avoidance" behavior (intention of exploring and communicating with others), and emotion responses (pleasure, arousal, and dominance). The questions addressed herein are as follows: a) What are the colors commonly used in the real public spaces of large shopping malls in China? What are the characteristics of color distribution? b) What are the effects of hue, saturation, and brightness on individual responses? c) Do the embodied and referential meanings of color have different effects on individual responses?

\section{Methodology}

To control the independent variables accurately, a laboratory experiment was conducted, in which audio-visual scenes of public spaces of shopping malls with controlled visual elements were played. Participants were invited to answer a corresponding questionnaire.

\subsection{Field survey}

At the beginning of this study, a field survey, involving seven shopping malls, was conducted to investigate the features of people's perception of the audio-visual environment in actual public spaces. To ensure diversity in interior decoration styles, the malls used for audio-visual analysis include various types: an international commercial chain (CapitaLand), a national commercial chain (Wanda), and a local commercial brand (Hongbo) in Harbin. At each type, images were taken, and sound recorded, with the results of both taken to the laboratory. All images were captured by a Nikon P7000 digital camera, and the sound recorded by a 10 -access sound signal collection system. A total of 150 questionnaires were distributed, in which participants were asked to select the audio and visual elements they noticed and then note their evaluation of the environment.

Results showed that the soundscape of the public space of shopping malls includes the voice of people, footsteps, music played by the broadcasting system, and background noise. To construct more realistic scenes in the experiment, typical sound clips were edited. According to the processing of the photos taken on-site, three visual elements 
appeared most frequently: store interfaces, large-scale advertisements, and guiding signs; the top three elements chosen by participants were store interfaces, large-scale advertisements, and people, indicating that these four visual elements are typical symbols of a shopping environment. By comparing the on-site photos and questionnaire results, each visual element's pixel proportion of the whole picture was calculated, as shown in Table 1; this provided the basis for the experimental materials, so as to avoid extreme situations and ensure typicality of the visual scenes.

Table 1

Pixel Proportion of the Whole Picture in the Visual Scenes of Typical Public Space of Shopping Malls.

\begin{tabular}{cccccccc}
\hline $\begin{array}{c}\text { Visual } \\
\text { Elements }\end{array}$ & $\begin{array}{c}\text { Store } \\
\text { Interface }^{\mathrm{a}}\end{array}$ & $\begin{array}{c}\text { Large-scale } \\
\text { Advertisement }^{\mathrm{a}}\end{array}$ & People & $\begin{array}{c}\text { Guiding } \\
\text { Signs }\end{array}$ & Chairs & Plants & Daylight \\
\hline $\begin{array}{c}\text { Pixel } \\
\text { Proportion }\end{array}$ & $16-22 \%$ & $2-4 \%$ & $2-4 \%$ & $0-4 \%$ & $0-4 \%$ & $0-10 \%$ & $0-6 \%$ \\
\hline $\begin{array}{l}\text { a Store interfaces and large-scale advertisements meet the above requirements respectively, or the } \\
\text { sum of the two is 17-25\% }\end{array}$
\end{tabular}

\subsection{Settings of the Scenes}

In the laboratory experiment, a total of 11 audio-visual scenes were shown to the participants. On weekends and holidays, some shopping malls hold events, such as special sales or talent shows. Thus, the 11th scene was a talent show held in a large atrium such that all possible activities in the public spaces of shopping malls were covered. Its audio-visual setting was not comparable to the others and was, therefore, excluded from subsequent analyses.

Before the experiment, the A-weighted equivalent continuous sound level (LAeq) of each sound signal collected on-site and edited was listened to and recorded by a dummy head. The LAeq of the playback sound was maintained within the range $65 \pm 3 \mathrm{~dB}(\mathrm{~A})$. [23]

\subsubsection{Selection and Adjustment of Pictures}

To get close to the real environment, the selection of pictures was based on the following principles. a) The content of pictures should conform to the vision field range of the human eyes, which is $60^{\circ}$ to the left and right, $60^{\circ}$ up, and $70^{\circ}$ down. The visual horizon should be at the normal level of sight for adults, i.e., 1.60 to $1.70 \mathrm{~m}$ high, and the images should be without perspective distortion. b) According to the results of the previous field survey (Table 1), the pixels' proportion of each visual element should be controlled. Figure 2 is an example of such a pixel control of visual elements in one of the experimental images. c) Various types of shopping malls should be represented, including the traditional department stores of the 1980s and 1990s, and the shopping centers that have emerged in the $21^{\text {st }}$ century. [24] d) All kinds of public spaces of shopping malls should be also covered, including large-open areas and narrow atriums, and both wide and narrow corridors. 


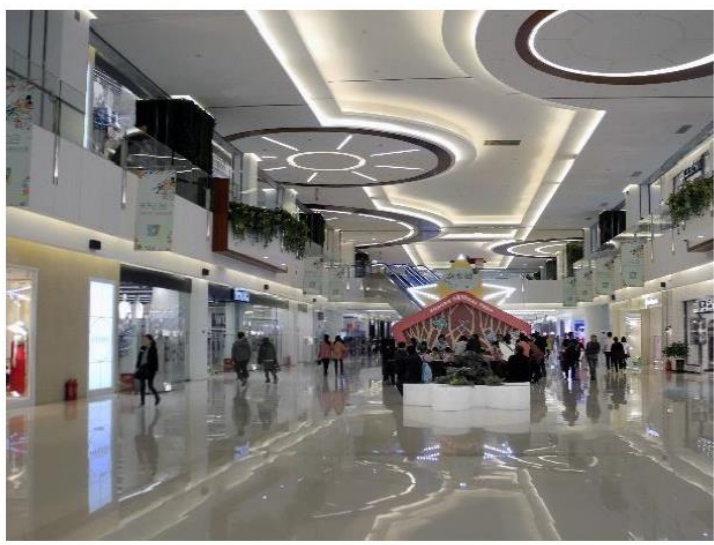

(A)

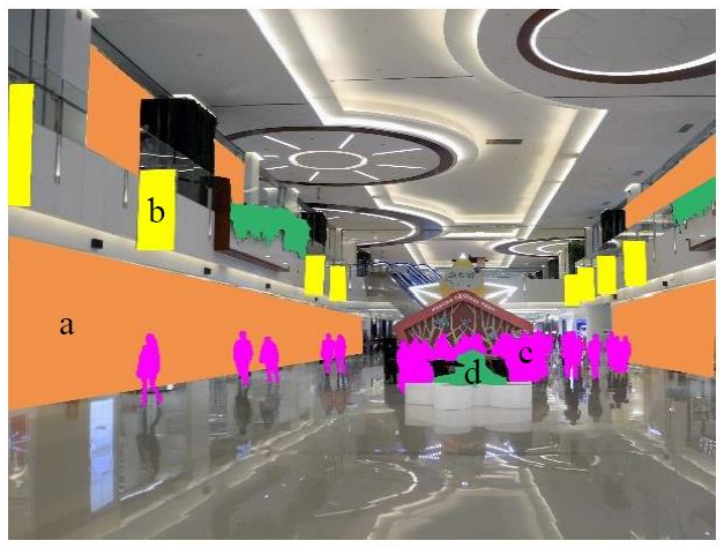

(B)

Fig. 2. Pixel Proportion Control of Images for the Experiment. A, Sample visual image used for the experiment. B, Pixel proportion of each visual element in the figure, "a" represents store interfaces $(14.37 \%)$, "b" advertisements $(2.82 \%)$, "c" people $(3.04 \%)$, and "d" plants $(1.52 \%)$.

There are two pairs of scenes in the experiment, in which red and green are selected as the research variables of the referential and embodied meanings of color, respectively. The green decorative belt in scene VCG1 corresponds to the green plants in scene VCG2; the red sale billboard in scene VCR1 corresponds to the red lanterns and carpet in scene VCR2 (Fig. 3).

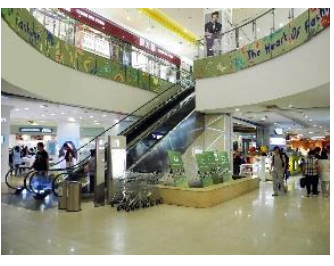

(A) VCG1: green decorative belt + display board (Green accounts for $10.01 \%$ pixels )

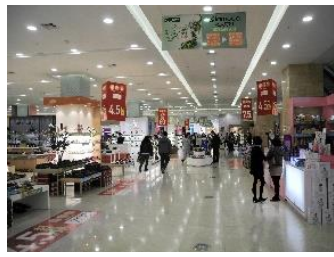

(C) VCR1: red billboard + advertising

(Red accounts for $17.99 \%$ pixels)


(B) VCG2: green plants

(Green accounts for $9.96 \%$ pixels )
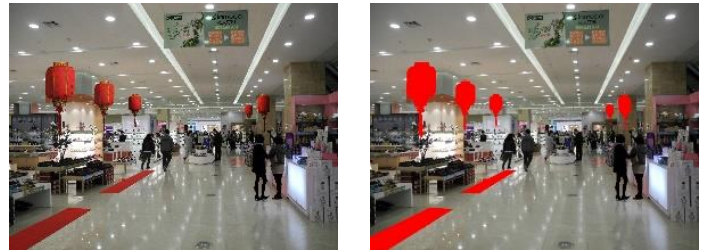

(D) VCR2: red lanterns + carpet

(Red accounts for $16.92 \%$ pixels)

Fig. 3. Variables of green and red color in the visual scene of the experiment. A, VCG1: green decorative belt + display board (green accounts for $10.01 \%$ pixels). B, VCG2: green plants (green accounts for $9.96 \%$ pixels). C, VCR1: red billboard + advertising (red accounts for $17.99 \%$ pixels). D, VCR2: red lanterns + carpet (red accounts for $16.92 \%$ pixels).

\subsubsection{Picture Processing}

To analyze the influence of color on individual responses, a color mode must be selected that can be extracted and processed by a computer for information quantization of the image colors. In Adobe Photoshop (PS), colors can be converted to hue-saturationbrightness (HSB), RGB, Lab, and cyan-magenta-yellow-black (CMYK) mode. Among them, the HSB mode is a color pattern based on the law of human visual perception. Therefore, this color mode was selected to process the visual images quantitatively. In the $\mathrm{HSB}$ mode, $\mathrm{H}$ is represented by an angle on the standard color ring of $0-360^{\circ}$; $\mathrm{S}$ 
represents the proportion of color components in a hue, measured as a percentage from $0 \%$ (grey) to $100 \%$ (fully saturated); B represents the brightness of a color, measured as a percentage from $0 \%$ (black) to $100 \%$ (white).

Images were processed using "Filter $\rightarrow$ Pixelate $\rightarrow$ Mosaic..." in the PS toolbar, where each pixel represented a single color. According to the minimum recognition size of the main elements in the visual scene, the mosaic cell size was determined to be $50 \times 50$ pixels. Comparing the original and the mosaic pictures, the differences in the overall $R G B$ value and that in the single color $R G B$ value were both $\leqslant 0.5$, indicating that "Mosaic" leads to very little change in a picture's colors, and the overall color of the images is restored at a very high level. Therefore, the processed images can represent the color of the original ones.

The "magic wand" tool in PS was used and the tolerance was set at the default value of 32. The H, S, and B values and the pixel number of each color were displayed through the built-in histogram tool of PS. Then, the pixels' proportion values were calculated. Main colors were extracted from each image, and the colors were named as first major color (MC1), second major color (MC2), and so on, according to the pixels proportion values in decreasing order. A color with a pixel proportion value less than $5 \%$ was considered as a minority color, and was removed, leaving 3 to 5 main colors of each scene.

According to the characteristics of the HSB value of the 39 colors extracted, taking into account the influence of the HSB value on the subjective effect of color presentation, the colors were classified mainly by saturation and brightness. When $\mathrm{S}<35 \%$ and $\mathrm{H}$ has no obvious trend, the brightness $B$ is considered as the classification standard, where each $20 \%$ interval is a classification. The following color classifications were obtained: 1 -dark (7 items), 2-dark greyish (8 items), 3-medium greyish (10 items), and 4-light greyish (6 items). Color classification 5-bluish (5 items), with a hue scope of $180^{\circ}$ to $240^{\circ}$, and color classification 6-reddish ( 3 items), with a hue scope of $0^{\circ}$ to $20^{\circ}$, are classified according to the features of hue (Table 2). Among them, there are 12 colors with a saturation less than or equal to $5 \%$, which are extremely close to grey colors; these accounted for over $30 \%$ of all the colors.

Table 2

Classification List of Colors Extracted from Visual Images (H:, S \&B: \%, Pixels Proportion (PP): \%).

\begin{tabular}{|c|c|c|c|c|c|c|c|c|c|c|}
\hline $\begin{array}{c}\text { Color } \\
\text { Classification } \\
\end{array}$ & V1 & V2 & V3 & V4 & V5 & V6 & VCG1 & VCG2 & VCR1 & VCR2 \\
\hline 1-Dark & & & & & & & & & & \\
\hline $\begin{array}{l}S \in(0,35) \\
B \in(10,30)\end{array}$ & $12 / 19 / 11$ & 0/2/19 & $52 / 25 / 23$ & $50 / 23 / 20$ & & $15 / 21 / 22$ & $240 / 1 / 27$ & & & $30 / 18 / 27$ \\
\hline $\mathrm{PP}$ & 11.43 & 10.24 & 35.59 & 31.52 & & 28.26 & 13.70 & & & 24.76 \\
\hline 2-Dark Greyish & & & & & & & & & & \\
\hline $\begin{array}{l}S \in(0,35) \\
B \in(30,50)\end{array}$ & $45 / 14 / 33$ & 9/7/38 & & $37 / 13 / 47$ & $22 / 14 / 31$ & $180 / 12 / 42$ & $64 / 30 / 41$ & $70 / 15 / 31$ & $45 / 5 / 33$ & \\
\hline $\mathrm{PP}$ & 28.00 & 10.13 & & 24.83 & 68.97 & & 11.72 & 29.51 & 31.42 & \\
\hline 3-Mid Greyish & & & & & & & & & & \\
\hline$S \in(0,35)$ & $37 / 16 / 56$ & $37 / 6 / 54$ & $48 / 26 / 52$ & $120 / 1 / 68$ & $327 / 5 / 65$ & $39 / 10 / 56$ & $62 / 17 / 62$ & $60 / 14 / 63$ & $44 / 10 / 61$ & $45 / 9 / 54$ \\
\hline
\end{tabular}




\begin{tabular}{|c|c|c|c|c|c|c|c|c|c|c|}
\hline $\mathrm{B} \in(50,70)$ & & & & & & & & & & \\
\hline $\mathrm{PP}$ & 54.92 & 77.86 & 36.32 & 37.42 & 30.82 & 49.23 & 57.96 & 55.85 & 54.41 & 67.82 \\
\hline \multicolumn{11}{|l|}{ 4-Light Greyish } \\
\hline $\begin{array}{l}S \in(0,35) \\
B \in(70.90)\end{array}$ & $48 / 3 / 78$ & $42 / 9 / 85$ & $60 / 7 / 76$ & & & & $60 / 3 / 81$ & $72 / 2 / 80$ & & $120 / 1 / 82$ \\
\hline $\mathrm{PP}$ & 5.60 & 5.53 & 31.20 & & & & 16.38 & 14.92 & & 5.39 \\
\hline \multicolumn{11}{|l|}{ 5-Bluish } \\
\hline $\begin{array}{c}H \in(180,240) \\
S \in(0,35)\end{array}$ & 222/17/62 & & & $204 / 2 / 97$ & & $180 / 3 / 79$ & $213 / 16 / 66$ & & $195 / 2 / 85$ & \\
\hline $\mathrm{PP}$ & 5.58 & & & 7.14 & & 11.00 & 5.53 & & 5.34 & \\
\hline \multicolumn{11}{|l|}{ 6-Redish } \\
\hline $\begin{array}{c}\mathrm{H} \in(0,20) \\
\mathrm{S} \in(30,100)\end{array}$ & & & & & & $4 / 47 / 45$ & & & $20 / 34 / 42$ & $7 / 68 / 57$ \\
\hline PP & & & & & & 8.72 & & & 14.78 & 6.09 \\
\hline
\end{tabular}

\subsection{Experimental Laboratory and Participants}

The experiment was conducted in the audiometric laboratory of a university in Harbin, China. The volume of the laboratory is $186 \mathrm{~m}^{3}$ and the background noise $11 \mathrm{~dB}(\mathrm{~A})$. A schematic diagram of the laboratory layout is shown in Figure 4. The images were displayed on a SAMSUNG 75-in. LCD television set (model number UA75F6400), which lets the participants watch with their full vision field; the audio was provided by an RME FireFace UFX sound card and a Genelec sound system, which creates a surround-sound effect deemed realistic for the sound recordings. Only the necessary equipment and no other objects were placed in the laboratory, such that the participants would be less likely to be distracted. [25]

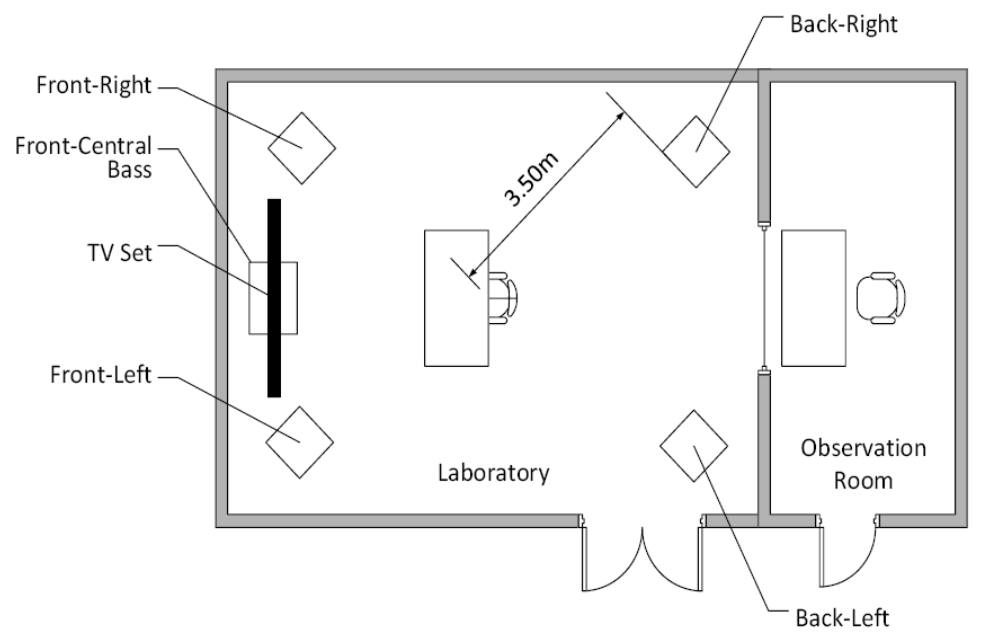

Fig. 4. Laboratory Layout Schematic.

A total of 70 randomly selected adults younger than 60 years participated in this experiment. Participants were recruited from among the postgraduates and staff members of a university and the staff of a public institution. They were informed by a volunteer recruitment notice through a social communication app. Informed consent was obtained from all participants, and all of them self-reported normal or corrected-to-normal hearing and vision. All participants were offered a small gift and some snacks as a token 
of appreciation after the experiment. All participants are native Chinese and have lived in Chinese culture most of their lives. Characteristics of the participants are presented in Table 3.

Table 3

Experiment Participants' Characteristics (Total participants $=70$ ).

\begin{tabular}{|c|c|c|c|c|c|c|c|}
\hline Gender & & Age & & Educational Background & & Occupatior & \\
\hline Male & 40 & $18-27$ & 23 & School/Junior & 9 & Students & 27 \\
\hline Female & 30 & $\begin{array}{l}28-44 \\
45-60\end{array}$ & $\begin{array}{l}30 \\
17\end{array}$ & $\begin{array}{l}\text { Undergraduate } \\
\text { Graduate } \\
\text { PhD }\end{array}$ & $\begin{array}{l}26 \\
21 \\
14\end{array}$ & $\begin{array}{l}\text { Teachers } \\
\text { Staff } \\
\text { Managers } \\
\text { Others }\end{array}$ & $\begin{array}{c}2 \\
19 \\
6 \\
16\end{array}$ \\
\hline
\end{tabular}

\subsection{Setting of the Questionnaire}

In 1957, Osgood et al. [26] introduced evaluation, activity, and potency as three factors of affective responses. In 1960, Rosenberg [27] developed the ABC psychological model, proposing that affect, behavior, and cognition are the three types of responses that occur after stimuli. Mehrabian and Russell [28] proposed the PAD three-dimensional emotion theory in 1974; according to this theory, all human emotions can be defined by three independent dimensions. Subsequently, many researchers conducted studies with this theory, and the PAD emotion theory was expanded and improved; it has now become one of the most important methods in the field of emotion study. [29] "Approachavoidance" behavior is the basic instinct of all living creatures, including humans; behavior characterized by approach avoidance often appears in the motivation, cognition, psychology, behavior, and other related studies. In the field of environmental psychology, the "approach-avoidance" behavior intention can be measured by choosing the degree of agreement between the feeling and the behavior descriptions that may occur $[13,15,28,30]$.

The questionnaire was divided into three parts. The first part pertained to shopping habits, which helped the participants visualize the shopping environment and become familiar with the laboratory. The second part was concerned with the participants' perception of the corresponding scenes, including the description of the behavior intention and adjectives related to the emotions, using a seven-point Likert scale and a seven-point semantic difference scale, [31] which is the core part of this study. The third part inquired about personal characteristics, including age, gender, educational background, and occupation.

Simple forward/reverse descriptive statements were used to obtain the participants' evaluation and behavior intention in the second part of the questionnaire. The subsequent dependent variables analysis involved satisfaction, approach-avoidance behavior, exploring behavior, communication with others, pleasure, arousal, and dominance. The details of the core part of the questionnaire are presented in Table 4. In the experiment, the questionnaire was written in Chinese.

Table 4 
Details of the Core Part of Questionnaire and Source of Scores for Dependent Variables.

\begin{tabular}{|c|c|c|c|c|c|c|c|c|c|c|c|c|c|c|c|c|c|c|c|}
\hline \multirow{4}{*}{ Satisfaction } & \multicolumn{19}{|c|}{ SCENE* } \\
\hline & & \multicolumn{18}{|c|}{ (1) Please read the following descriptions, draw $\sqrt{ }$ at the most suitable position according to your actual feelings. } \\
\hline & \multicolumn{10}{|c|}{ I am very satisfied with the environment of the store. } & Totally agree & 1 & 2 & 3 & 4 & 5 & 6 & 7 & Totally disagree \\
\hline & & \multicolumn{9}{|c|}{ I like this environment. } & Totally agree & 1 & 2 & 3 & 4 & 5 & 6 & 7 & Totally disagree \\
\hline \multirow{3}{*}{$\begin{array}{c}\text { Approach-avoidance } \\
\text { Behavior }\end{array}$} & \multicolumn{10}{|c|}{ I might not come to this store any more. } & Totally agree & 1 & 2 & 3 & 4 & 5 & 6 & 7 & Totally disagree \\
\hline & & \multicolumn{9}{|c|}{ I might stay here longer than planned. } & Totally agree & 1 & 2 & 3 & 4 & 5 & 6 & 7 & Totally disagree \\
\hline & & \multicolumn{9}{|c|}{ I would like to take some time to browse the store. } & Totally agree & 1 & 2 & 3 & 4 & 5 & 6 & 7 & Totally disagree \\
\hline \multirow{2}{*}{$\begin{array}{l}\text { Exploring } \\
\text { Behavior }\end{array}$} & & \multicolumn{9}{|c|}{ I would like to shop at this store. } & Totally agree & 1 & 2 & 3 & 4 & 5 & 6 & 7 & Totally disagree \\
\hline & \multicolumn{10}{|c|}{ I want to get out of this store as soon as possible. } & Totally agree & 1 & 2 & 3 & 4 & 5 & 6 & 7 & Totally disagree \\
\hline \multirow{2}{*}{$\begin{array}{l}\text { Communication } \\
\text { With Others }\end{array}$} & & \multicolumn{9}{|c|}{ I will chat with other customers I meet here. } & Totally agree & 1 & 2 & 3 & 4 & 5 & 6 & 7 & Totally disagree \\
\hline & & \multicolumn{9}{|c|}{ I will try to hide from others and not talk to strangers. } & Totally agree & 1 & 2 & 3 & 4 & 5 & 6 & 7 & Totally disagree \\
\hline & & \multicolumn{18}{|c|}{ (2) Please read the following opposite phrases, draw $\sqrt{ }$ on the most suitable position according to your actual feelings. } \\
\hline Emotion & & \multirow{3}{*}{$\begin{array}{c}\text { Satisfied } \\
\text { Comfortable } \\
\text { Fevered }\end{array}$} & 1 & 2 & 3 & 4 & 5 & 6 & 7 & Depressed & Happy & 1 & 2 & 3 & 4 & 5 & 6 & 7 & Unhappy \\
\hline Pleasure & & & 1 & 2 & 3 & 4 & 5 & 6 & 7 & Restless & Glad & 1 & 2 & 3 & 4 & 5 & 6 & 7 & Angry \\
\hline \multirow{2}{*}{ Arousal } & & & 1 & 2 & 3 & 4 & 5 & 6 & 7 & Peaceful & Excited & 1 & 2 & 3 & 4 & 5 & 6 & 7 & Unexcited \\
\hline & & Incitant & 1 & 2 & 3 & 4 & 5 & 6 & 7 & Relaxed & Awakened & 1 & 2 & 3 & 4 & 5 & 6 & 7 & Drowsy \\
\hline Dominance & & Active & 1 & 2 & 3 & 4 & 5 & 6 & 7 & Passive & Uncontrolled & 1 & 2 & 3 & 4 & 5 & 6 & 7 & Controlled \\
\hline
\end{tabular}

\subsection{Experimental Process}

The experimental process is shown in Figure 5. At the beginning of the experiment, the participants entered the laboratory under the guidance of the experimenter and then sat at their designated positions. The experimental process and operational essentials were introduced to the participants. Each audio-visual scene was played for 30 seconds [22, $32,33,35,36]$. The main part of the experiment was controlled to last approximately 20 minutes, to avoid errors due to fatigue. After the experiment, approximately 3 minutes were allotted to the participants to communicate with the experimenter about their feelings and experience. To reduce the influence of additional visual stimulus variables on the experimental results, the background color of the experimental program was set as grey $(R=75, G=75, B=75)$, and the text was white $(R=255, G=255, B=255)$. The experimenter wore low-saturation colors with light or no makeup.

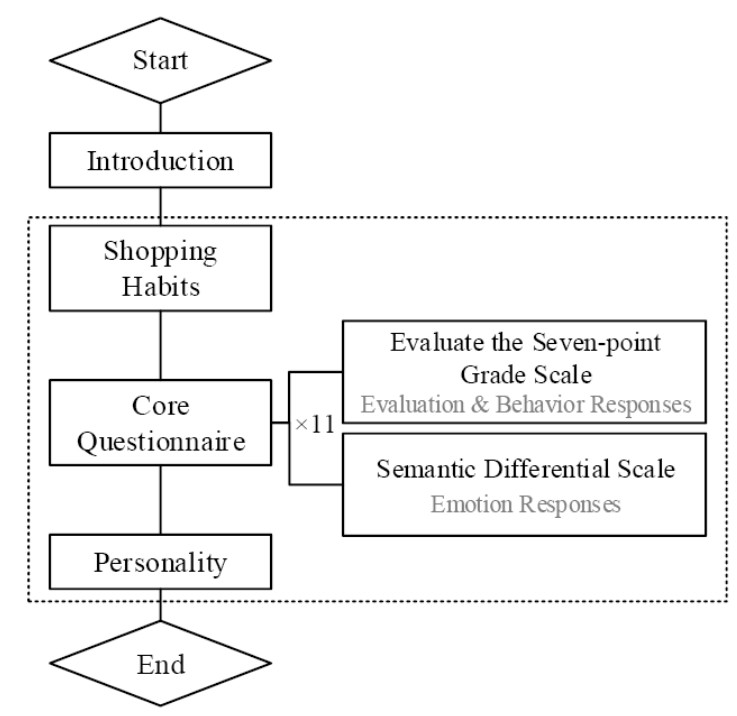

Fig. 5. Experimental Flow Diagram.

\subsection{Statistical Analysis Method}

In this study, analysis of variance (ANOVA) was used to test the significance of difference between the means of two or more categories of variables. For the variables with significant differences, multiple comparison was further used to determine which level of 
the independent variable had a significant effect on the dependent variable. In the homogeneity test of variance, when sig. was greater than .05, which was consistent with the homogeneity of variance hypothesis, the LSD method was used for multiple comparison analysis. And when sig. was less than .05, which was not consistent with the homogeneity hypothesis, Tamhane's T2 method was used. The influence of four groups of hues on individual responses was tested by ANOVA and multiple comparison. The $p$ value was corrected by Bonferroni correction method $(p=.05 / 6=.00833)$.

Correlation analysis is used to compare the relationship between two groups of continuous numerical variables. When the analysis result $p$ value is less than 0.05 , the null hypothesis is rejected, indicating that there is a correlation between the two variables. The influences of saturations and brightness differences on individual responses were tested by correlation analysis method.

The paired sample $t$ test was used between two pairs of variables when the study considers the embodied meaning and referential meaning of colors, to test whether there are significant differences between the data of the two green scenes and the two red scenes.

The dependent and independent variables used herein are presented in Table 5.

Table 5

Dependent and Independent Variables.

\begin{tabular}{|c|c|c|c|c|}
\hline $\begin{array}{l}\text { Statistical } \\
\text { Analysis }\end{array}$ & \multicolumn{3}{|c|}{ Independent Variables } & Dependent Variables \\
\hline \multirow{4}{*}{ ANOVA } & \multirow{4}{*}{$\begin{array}{c}\mathrm{H} \\
\text { (Independent } \\
\text { Discrete } \\
\text { Nominal) }\end{array}$} & $\mathrm{H}_{\mathrm{G}}$ & $\begin{array}{l}\text { Hue of scenes without bluish and } \\
\text { reddish colors }\end{array}$ & \multirow{4}{*}{ Satisfaction } \\
\hline & & $\mathrm{H}_{\mathrm{G}+5}$ & Hue of scenes with bluish color & \\
\hline & & $\mathrm{H}_{\mathrm{G}+6}$ & Hue of scenes with reddish color & \\
\hline & & $\mathrm{H}_{\mathrm{G}+5+6}$ & $\begin{array}{l}\text { Hue of scenes with both bluish } \\
\text { and reddish colors }\end{array}$ & \\
\hline \multirow{5}{*}{$\begin{array}{l}\text { Correlation } \\
\text { Analysis }\end{array}$} & \multirow{2}{*}{$\begin{array}{c}\text { S } \\
\text { (Independent } \\
\text { Continuous } \\
\text { Scale) }\end{array}$} & $\begin{array}{c}\mathrm{S} \\
\mathrm{S}_{\mathrm{MC} 1} \\
\end{array}$ & $\begin{array}{l}\text { Average saturation of each scene } \\
\text { Saturation of the first main color }\end{array}$ & $\begin{array}{c}\text { Approach-avoidance } \\
\text { Behavior }\end{array}$ \\
\hline & & $\mathrm{Scc3}$ & Saturation of color classification 3 & Exploring Behavior \\
\hline & \multirow{3}{*}{$\begin{array}{c}\text { B } \\
\text { (Independent } \\
\text { Continuous } \\
\text { Scale) }\end{array}$} & $\Delta \mathrm{B}_{12}$ & $\begin{array}{l}\text { Brightness difference between } \\
\text { the first and second main colors } \\
\text { of each scene }\end{array}$ & \multirow{4}{*}{$\begin{array}{c}\text { Communication } \\
\text { Pleasure } \\
\text { Arousal } \\
\text { Dominance } \\
\text { (Independent } \\
\text { Continuous } \\
\text { Scale) }\end{array}$} \\
\hline & & $\Delta \mathrm{B}_{13}$ & $\begin{array}{l}\text { Brightness difference between } \\
\text { the first and third main colors of } \\
\text { each scene }\end{array}$ & \\
\hline & & $\Delta \mathrm{B}_{23}$ & $\begin{array}{l}\text { Brightness difference between } \\
\text { the second and third main colors } \\
\text { of each scene }\end{array}$ & \\
\hline $\begin{array}{l}\text { Paired } \\
\text { Sample } \\
\text { T-test }\end{array}$ & \multicolumn{2}{|c|}{$\begin{array}{l}\text { VCR1\&VCG2 } \\
\text { VCG1\&VCG2 } \\
\text { (Two Pairs of } \\
\text { Variables) }\end{array}$} & $\begin{array}{c}\text { Two pairs of scenes containing } \\
\text { different items with same color } \\
\text { red or green }\end{array}$ & \\
\hline
\end{tabular}

\section{Results}

\subsection{Color Distribution Characteristics}


The color distribution characteristics of the visual scene are shown in Figure 6. First, in terms of hue, 29 colors (approximately $74.3 \%$ ) out of the total 39 colors, are distributed within the range $0-80^{\circ}$, as the colors red, orange, and yellow; $17.9 \%$ of the colors' (corresponding to seven items) hues are distributed within the range $180^{\circ}$ to $240^{\circ}$, as blue. It is observed that in the common public spaces of shopping malls, the main color hue is a warm color, matched with a part of blue color. The frequency of other colors is very low.

Second, the saturation of $92.3 \%$ of colors is less than $33 \%$, which belongs to the greyish color series. The saturation of $5.1 \%$ of colors, is within $34 \%$ and $66 \%$, which belongs to the medium saturation range. Only one color ( $2.6 \%$ of colors) has a high saturation, but the saturation is $68 \%$, which is very close to the medium saturation range and accounts for a very low proportion of pixels. This indicates that in the common public spaces of shopping malls, there are few colors with high saturation. Even if they exist, high saturation colors do not appear over a large area. The saturation of most colors is maintained at a very low level, and the overall color is mainly a greyish color.

Third, it can be observed from Figure 6 that the overall brightness range of all the scenes is within $11 \%$ to $97 \%$, which spans across almost all brightness ranges and is evenly distributed. The average brightness of each scene has little difference: the lowest is $42 \%$ and the highest is $58 \%$. Thus, in the common public spaces of shopping malls, the overall brightness of colors is relatively balanced, and the colors of dark, bright, and moderate brightness exist simultaneously.

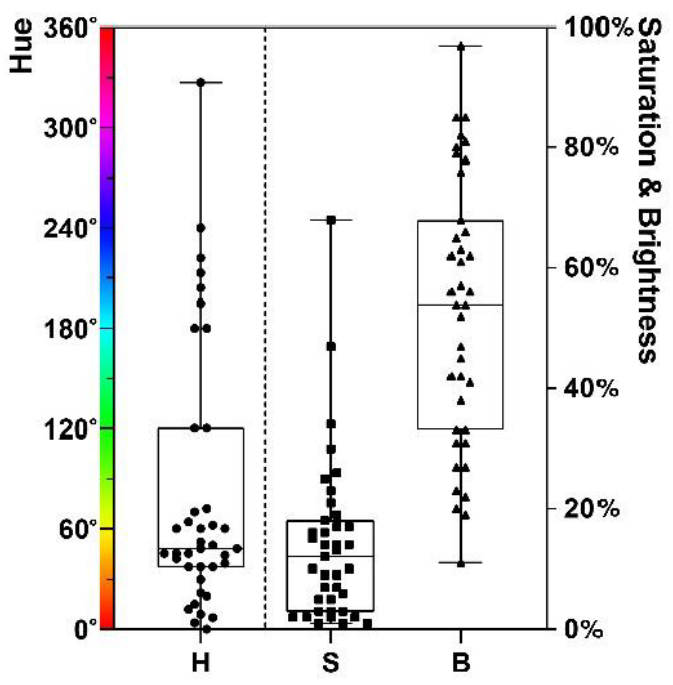

Fig. 6. Color Distribution of all Visual Scenes.

As observed in Table 2, every scene contains black or dark greyish, medium greyish, and light greyish colors or blue, indicating that although each scene contains different visual elements, the overall color composition is similar. In all color classifications, classification 3 , the mid-greyish color, is the most widely distributed and exists in all 10 scenes, followed by classifications 2 and 4 .

Chi-square tests were conducted on the composition of color classifications of the first and second main color in each scene, and the main colors showed a significance level of 0.01 for the color classifications (chi $=13.400, p=.004<.01$ ). The first main color of 
each scene is mainly composed of mid-greyish, accounting for $90 \%$, while only one scene's first main color is composed of classification 2. The second main color of all scenes is composed of four colors: $80 \%$ of which are dark colors, among which classification 1 has the highest proportion (accounting for half), and classification 2 takes the second place (accounting for 30\%). The third main color of all the scenes is composed of five colors, and the distribution is relatively balanced. This indicates that in each scene, the colors that occupy the largest proportion of the images are very similar; there is no obvious difference until the color that occupies the third largest proportion of the images. The color classification of the top three main colors of each scene is shown in Figure 7.

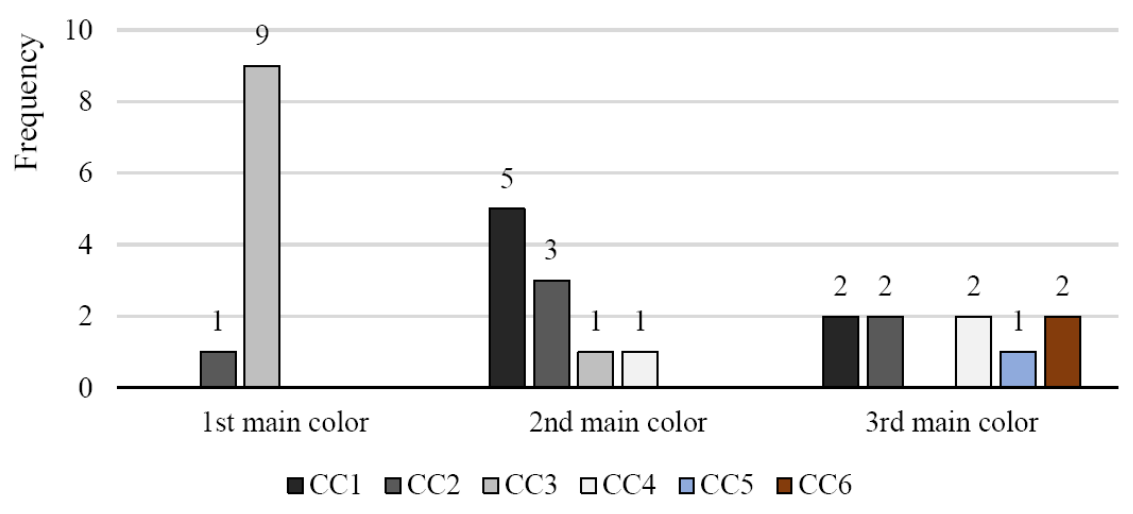

Fig. 7. Frequency of Color Classifications (CC) of Top 3 Main Colors in each Visual Scene. CC1 to CC6 correspond to CCs 1 to 6 of Table 2, respectively.

\subsection{Color and Individual Responses}

\subsubsection{Hue}

In the 39 colors of the six color classifications extracted from the visual images, although the hues of classifications 1 to 4 cover almost $90 \%$ degrees, they belong to a low saturation color, as their S values are lower than 35\%. The impact of color hue on a person's subjective feeling is limited; thus, these four types of color classifications belong to the greyish colors, the hue values of which are represented by " $\mathrm{H}_{\mathrm{G}}$." $\mathrm{H}_{\mathrm{G}}$ plus the hues of colors which belong to classification 5 are denoted as " $\mathrm{H}_{\mathrm{G}+5}$," in addition to those that

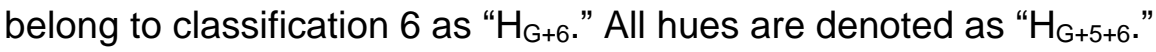

The data were subjected to a mixed-design ANOVA of all the individual responses $\times$ hue classifications. Some of the individual responses have a significance value less than .05 (Table 6).

\section{Table 6}

One Way ANOVA of Individual Responses $\times$ Hue Classifications.

\begin{tabular}{cccc}
\hline Individual Responses & Satisfaction & Approach-avoidance Behavior & Arousal \\
\hline $\mathrm{F}(3,556)$ & 6.864 & 5.453 & 6.367 \\
$p$ & & $<.001$ & \\
$\eta^{2} \mathrm{p}$ & 0.036 & 0.029 & 0.033 \\
\hline
\end{tabular}

Note. $p<.05$ are in boldface. 
Multiple comparisons indicate that the satisfaction increases between $\mathrm{H}_{\mathrm{G}+5}$ and $\mathrm{H}_{\mathrm{G}}(p$ $=.006)$ as well as between $\mathrm{H}_{\mathrm{G}+6}$ and $\mathrm{H}_{\mathrm{G}}(p<.001)$. The "approach-avoidance" behavior increases between $\mathrm{H}_{\mathrm{G}+6}$ and $\mathrm{H}_{\mathrm{G}}(p=.002)$. For emotion responses, arousal increases between $\mathrm{H}_{\mathrm{G}}$ and $\mathrm{H}_{\mathrm{G}+5+6}(p<.001)$. There is no significant difference in other situations.

Hue classification has a slight effect on individual responses. The relationship between hue classification and individual responses is shown in Figure 8. First, for the satisfaction and behavior intention, the evaluation of the scenes with blue and red colors is significantly lower than that of the scenes without these two colors. Second, in terms of emotion, compared with the scene with only greyish, the arousal increases in the "greyish + blue + red" scene significantly.
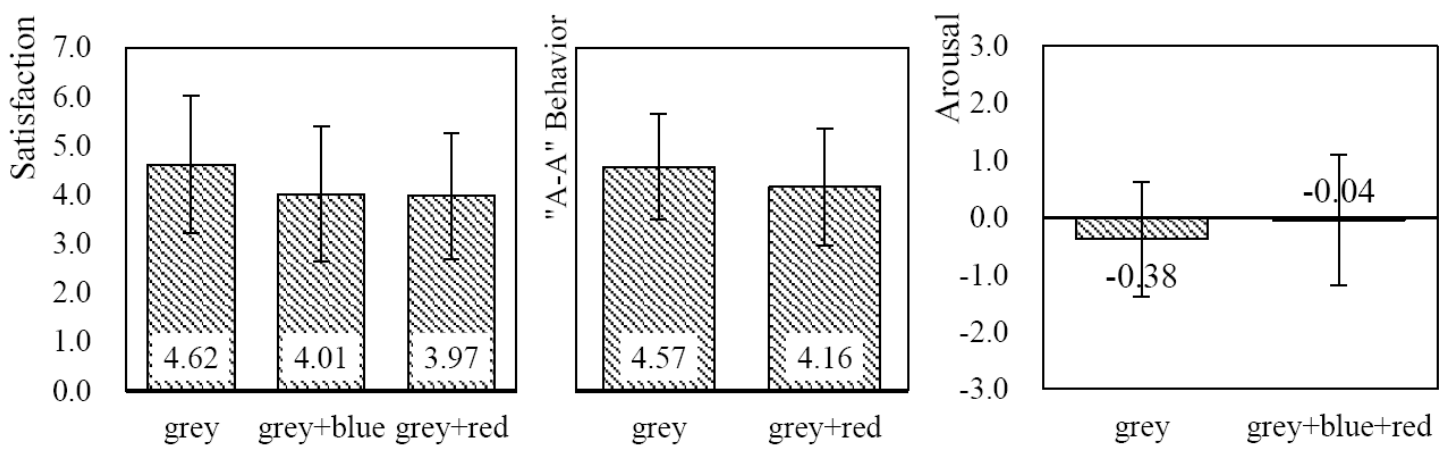
a) Satisfaction
b) "Approach- Avoidance" Behavior
c) Arousal

Fig. 8. Hue Classfication and Individual Responses.

\subsubsection{Saturation}

Using correlation analysis, the influence of saturation (S) of each visual scene, the saturation of the first main color $\left(\mathrm{S}_{\mathrm{MC} 1}\right)$, and the saturation of color classification $3\left(\mathrm{~S}_{\mathrm{CC} 3}\right)$ on individual responses were analyzed. The correlation analysis results are shown in Table 7. As can be seen in Table 7, all individual responses except for arousal have a significance value less than .05. Hypothesis testing one reveals that the difference in the individual responses, except for arousal, based on the influence of S, SMC1, and SCC3 is significant.

Table 7

Correlation Analysis of Saturation and Individual Responses.

\begin{tabular}{|c|c|c|c|c|c|c|c|c|}
\hline & Correlation & Satisfaction & $\begin{array}{l}\text { "Approach- } \\
\text { avoidance" } \\
\text { Behavior }\end{array}$ & $\begin{array}{l}\text { Exploring } \\
\text { Behavior }\end{array}$ & Communication & $P$ & A & $\mathrm{D}$ \\
\hline \multirow{3}{*}{$S$} & $\begin{array}{l}\text { Correlation } \\
\text { Coefficient }\end{array}$ & -.264 & -.232 & -.247 & -.111 & -.224 & .052 & -.199 \\
\hline & Sig. (2-tailed) & & $<.001$ & & .003 & $<.001$ & .169 & $<.001$ \\
\hline & $\eta$ & 0.315 & 0.327 & 0.316 & 0.146 & 0.307 & 0.190 & 0.251 \\
\hline \multirow{3}{*}{$\mathrm{S}_{\mathrm{MC} 1}$} & $\begin{array}{l}\text { Correlation } \\
\text { Coefficient }\end{array}$ & -.219 & -.193 & -.199 & -.084 & -.199 & -.031 & -.173 \\
\hline & Sig. (2-tailed) & & $<.001$ & & .026 & $<.001$ & .417 & $<.001$ \\
\hline & $\eta$ & 0.270 & 0.352 & 0.291 & 0.151 & 0.298 & 0.204 & 0.237 \\
\hline $\mathrm{Scc3}$ & $\begin{array}{l}\text { Correlation } \\
\text { Coefficient }\end{array}$ & -.222 & -.197 & -.200 & -.101 & -.214 & .063 & -.182 \\
\hline
\end{tabular}


Sig. (2-tailed)

$<.001$

.007

$<.001 \quad .097<.001$

$\eta$

0.278

0.335

0.289

0.154

$0.316 \quad 0.195$

0.243

Note. Sig. $<.05$ are in boldface.

The regression analysis of the influence of saturation on individual responses is shown in Figure 9. When the saturations $\left(\mathrm{S}, \mathrm{S}_{\mathrm{MC} 1}\right.$ and $\left.\mathrm{S}_{\mathrm{CC} 3}\right)$ are less than $10 \%$, the evaluations increase as the saturations decrease. Each evaluation reaches a low level when the saturation is higher than $10 \%$. When the saturations are between $10 \%$ and $16 \%$, the responses are basically unchanged, such that the evaluation values of satisfaction, "approach-avoidance" behavior, and exploring behavior are all approximately 4.5, and the emotion responses ( $P$ and $D)$ are approximately 0.75 . When the saturations are at the highest value, the evaluation values rebound. In addition, the organism action of behavior responses changes more sensitively with the increase and decrease of the overall saturation, whereas the language communication change is relatively gentle.
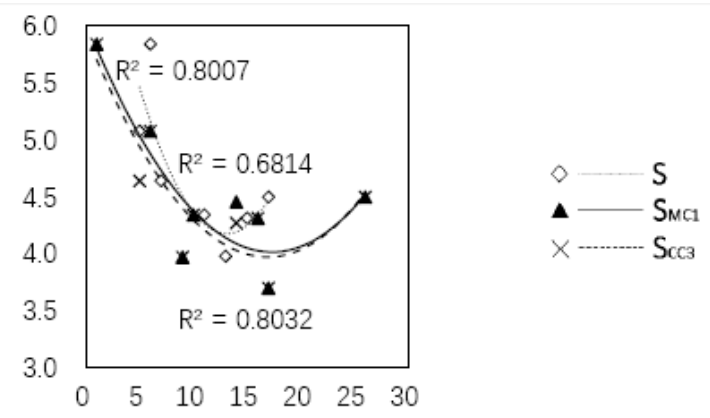

Satisfaction
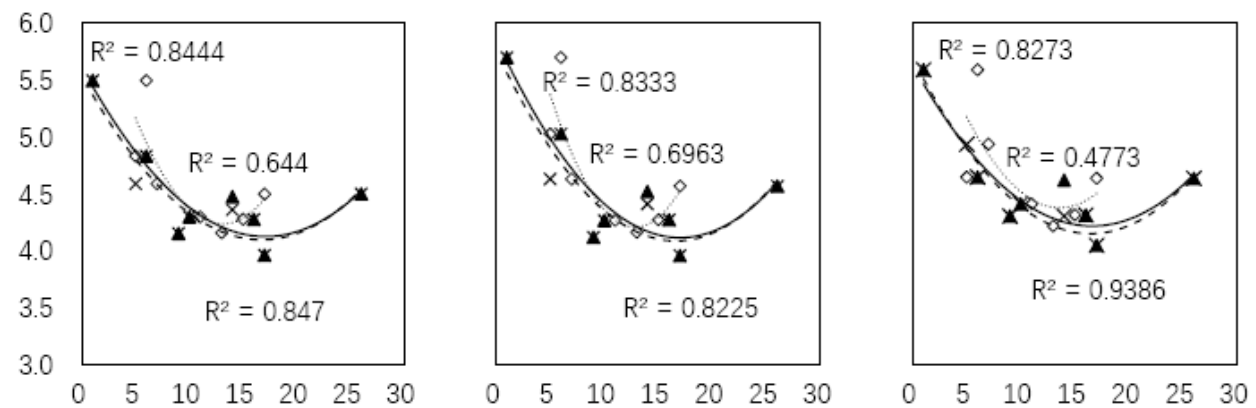

"Approach- Avoidance"

Exploring Behavior

Communication with others

Behavior
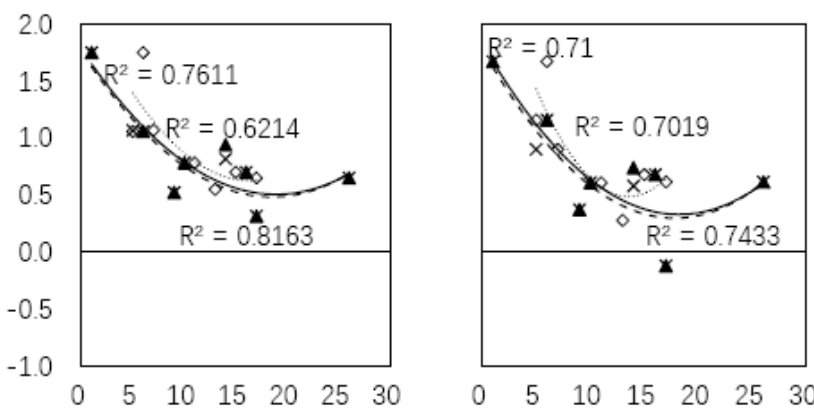

Pleasure

Dominance

Fig. 9. Saturation and Individual Responses.

Color Research and Application, Volume 45, 2020, 15 pages 


\subsubsection{Brightness}

It was observed that the average brightness range of each scene is relatively concentrated. The difference in brightness between the main colors in a scene is mainly considered to explore the influence of color brightness in a visual scene on individual responses. Therefore, the brightness differences between the first, second, and third main colors in a scene are denoted by the following: $\Delta \mathrm{B}_{12}, \Delta \mathrm{B}_{13}$, and $\Delta \mathrm{B}_{23}$, respectively. The results are shown in Table 8.

Table 8

Color Brightness Difference of Each Visual Scene (\%).

\begin{tabular}{ccccccccccc}
\hline Scenes & V1 & V2 & V3 & V4 & V5 & V6 & VCG1 & VCG2 & VCR1 & VCR2 \\
\hline$\Delta \mathrm{B}_{12}$ & 23 & 35 & 29 & 48 & 34 & 34 & 19 & 32 & 28 & 27 \\
$\Delta \mathrm{B}_{13}$ & 45 & 16 & 24 & 21 & 61 & 23 & 35 & 17 & 19 & 3 \\
$\Delta \mathrm{B}_{23}$ & 22 & 19 & 53 & 27 & 27 & 57 & 54 & 51 & 9 & 30 \\
\hline
\end{tabular}

Correlation analysis was conducted on the brightness differences and individual responses (Table 9). All individual responses have a significance value less than .05; hypothesis testing one reveals that the difference in the individual responses based on $\Delta \mathrm{B}_{12}$ is significant. The individual responses have a significance value greater than .05; hypothesis testing one reveals that the difference in the individual responses based on $\Delta \mathrm{B}_{13}$ is not significant. Individual responses, except for "Communication," have a significant value less than .05 ; hypothesis testing one reveals that the difference in the individual responses based on $\Delta \mathrm{B}_{23}$ is significant.

Table 9

Correlation Analysis of $\Delta \mathrm{B}_{12} 、 \Delta \mathrm{B}_{13} 、 \Delta \mathrm{B}_{23}$ and Individual Responses.

\begin{tabular}{|c|c|c|c|c|c|c|c|c|}
\hline & Correlation & Satisfaction & $\begin{array}{c}\text { "Approach- } \\
\text { avoidance" } \\
\text { Behavior }\end{array}$ & $\begin{array}{l}\text { Exploring } \\
\text { Behavior }\end{array}$ & Communication & $P$ & A & D \\
\hline \multirow{3}{*}{$\Delta \mathrm{B}_{12}$} & $\begin{array}{l}\text { Correlation } \\
\text { Coefficient }\end{array}$ & .357 & .322 & .336 & .154 & .295 & .088 & .262 \\
\hline & Sig. (2-tailed) & & & $<.001$ & & & .020 & $<.001$ \\
\hline & $\eta$ & 0.401 & 0.416 & 0.404 & 0.225 & 0.383 & 0.226 & 0.291 \\
\hline \multirow{3}{*}{$\Delta \mathrm{B}_{13}$} & $\begin{array}{l}\text { Correlation } \\
\text { Coefficient }\end{array}$ & -.008 & -.014 & -.019 & .019 & .015 & .019 & -.002 \\
\hline & Sig. (2-tailed) & .833 & .703 & .619 & .615 & .692 & .620 & .951 \\
\hline & $\eta$ & 0.123 & 0.227 & 0.167 & 0.126 & 0.201 & 0.221 & 0.097 \\
\hline \multirow{3}{*}{$\Delta \mathrm{B}_{23}$} & $\begin{array}{l}\text { Correlation } \\
\text { Coefficient }\end{array}$ & -.112 & -.094 & -.090 & -.064 & -.085 & .132 & -.135 \\
\hline & Sig. (2-tailed) & .003 & .013 & .017 & .089 & .024 & \multicolumn{2}{|c|}{$<.001$} \\
\hline & $\eta$ & 0.205 & 0.228 & 0.190 & 0.112 & 0.194 & 0.198 & 0.197 \\
\hline
\end{tabular}

Note. Sig. $<.05$ are in boldface.

The relationship between brightness difference between the first and second main colors $\left(\Delta \mathrm{B}_{12}\right)$, second and third main colors $\left(\Delta \mathrm{B}_{23}\right)$, and individual responses is shown in Figure 10. The individual responses are significantly positively correlated with $\Delta \mathrm{B}_{12}$, indicating that when the brightness difference between colors of the two largest areas of a scene increases, people's satisfaction and approach behavior increase, such that they have a stronger desire to further explore and interact with others. Simultaneously, emotional 
responses of pleasure and dominance improve as $\Delta \mathrm{B}_{12}$ increases. In terms of arousal, the evaluation increases with the increase in $\Delta \mathrm{B}_{12}$, indicating that the stronger the brightness contrast between the two colors with the largest proportion of pixels, the higher the arousal degree of the participants and, hence, the more positive they feel about the environment.

The trend of the effect of $\Delta \mathrm{B}_{23}$ on all individual responses except for arousal is very similar. All of them have the highest evaluation when the brightness difference is approximately $30 \%$, and the evaluation is low when the brightness difference is lower or higher than $30 \%$. However, the individual responses under the influence of $\Delta \mathrm{B}_{23}$ are more scattered, and the value of the goodness-of-fit judgment coefficient $R^{2}$ is small, whose rule is not as strong as the rule that $\Delta \mathrm{B}_{12}$ affects the individual responses. Additionally, $\Delta \mathrm{B}_{23}$ has a significant effect on arousal, which increases with the increase in brightness difference.

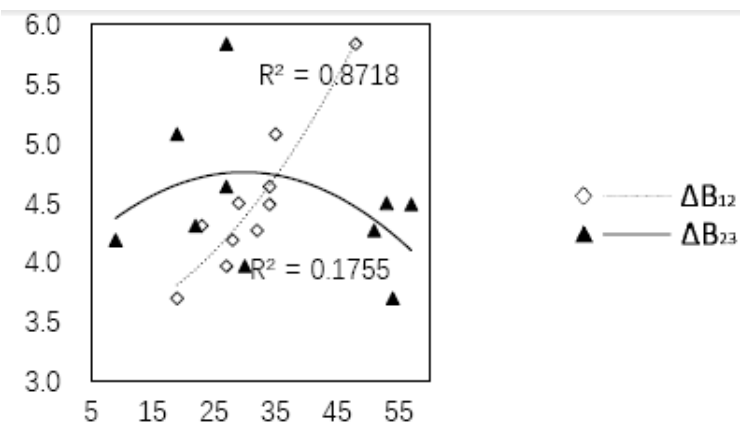

Satisfaction

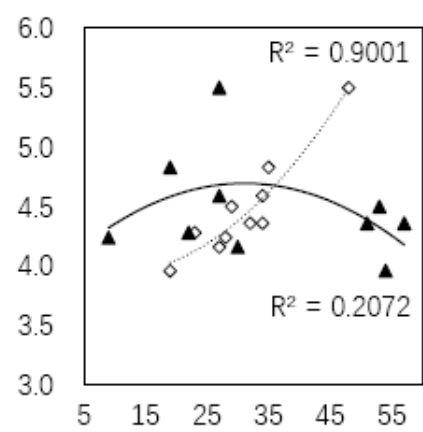

"Approach- Avoidance"

Behavior

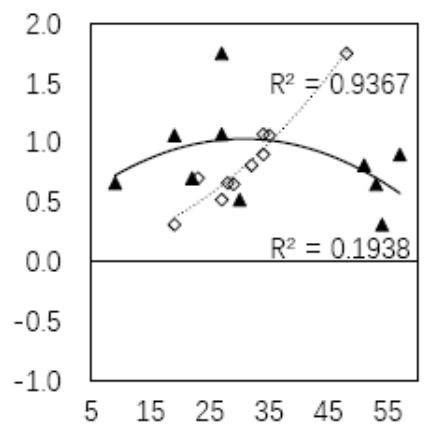

Pleasure

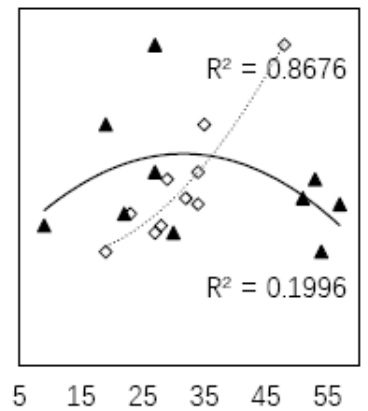

Exploring Behavior

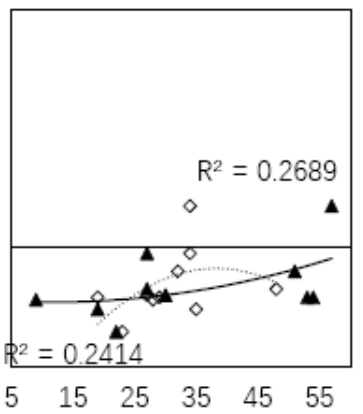

Arousal

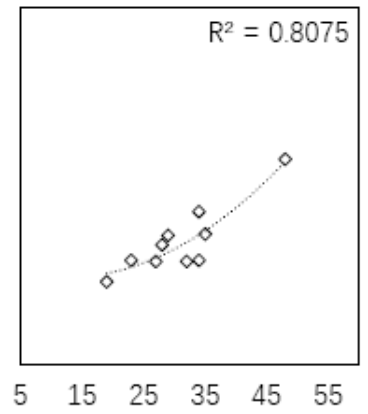

Communication with others

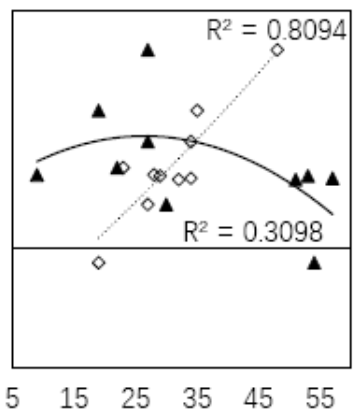

Dominance

Fig. 10. $\Delta \mathrm{B}_{12}, \Delta \mathrm{B}_{23}$ and Individual Responses.

Color Research and Application, Volume 45, 2020, 15 pages 


\subsubsection{Referential and Embodied Meanings}

The paired sample $t$ test of relevant samples was conducted on the data of the difference in the influence of the referential and embodied meanings of color on individual responses between the two green scenes and the two red scenes. The results are shown in Table 10.

Table 10

Paired Sample T-test of Color Meaning.

\begin{tabular}{|c|c|c|c|c|c|c|c|c|c|}
\hline Scene & $\begin{array}{r}\text { Statistice } \\
\text { Ite }\end{array}$ & $\begin{array}{l}\text { Analysis } \\
\mathrm{ns}\end{array}$ & Satisfaction & $\begin{array}{l}\text { "Approach- } \\
\text { avoidance" } \\
\text { Behavior }\end{array}$ & $\begin{array}{l}\text { Exploring } \\
\text { Behavior }\end{array}$ & Communication & $P$ & $A$ & $\mathrm{D}$ \\
\hline \multirow{4}{*}{$\begin{array}{l}\text { VCG1 } \\
\& \\
\text { VCG2 }\end{array}$} & $\begin{array}{l}\text { Paired } \\
\text { Sample }\end{array}$ & $\begin{array}{l}\text { Correlation } \\
\text { Coefficient }\end{array}$ & 0.402 & 0.495 & 0.357 & 0.585 & 0.385 & 0.327 & 0.34 \\
\hline & Correlation & $p$ & 0.001 & 0.000 & 0.002 & 0.000 & 0.001 & 0.006 & 0.004 \\
\hline & $\begin{array}{l}\text { Paired } \\
\text { Sample }\end{array}$ & $\begin{array}{c}\text { Sig. } \\
\text { (2-tailed) }\end{array}$ & .002 & .003 & .005 & .297 & .002 & .074 & .001 \\
\hline & Difference & $\mathrm{t}$ & -3.158 & -3.114 & -2.909 & -1.051 & -3.148 & -1.816 & -3.340 \\
\hline \multirow{4}{*}{$\begin{array}{l}\text { VCR1 } \\
\& \\
\text { VCR2 }\end{array}$} & $\begin{array}{l}\text { Paired } \\
\text { Sample }\end{array}$ & $\begin{array}{l}\text { Correlation } \\
\text { Coefficient }\end{array}$ & 0.42 & 0.392 & 0.286 & 0.607 & 0.314 & 0.456 & 0.333 \\
\hline & Correlation & $p$ & 0.000 & 0.001 & 0.016 & 0.000 & 0.008 & 0.000 & 0.005 \\
\hline & $\begin{array}{l}\text { Paired } \\
\text { Sample }\end{array}$ & $\begin{array}{l}\text { Sig. } \\
\text { (2-tailed) }\end{array}$ & .221 & .580 & .751 & .421 & .439 & .791 & .259 \\
\hline & Difference & $t$ & -1.235 & -0.556 & -0.318 & -0.809 & -0.778 & 0.266 & -1.139 \\
\hline
\end{tabular}

Note. $p<.05$ are in boldface.

It is observed from Table 10 that different colors affect individual responses with different meanings in an actual scene. However, in the two green scenes, there is a significant difference in the individual responses (except for that regarding the communication with others and arousal), and the significance level is always less than .05 . The green color of VCG1 is for the green decorative belt and that of VCG2 is for the green plants. As shown in Figure 11, the evaluations of VCG2 are all higher than those of VCG1, indicating that in the public spaces of shopping malls, green mainly shows referential meaning. The greenery brings a positive experience not because of the meaning of the color itself but because of the "plant" properties of the material.

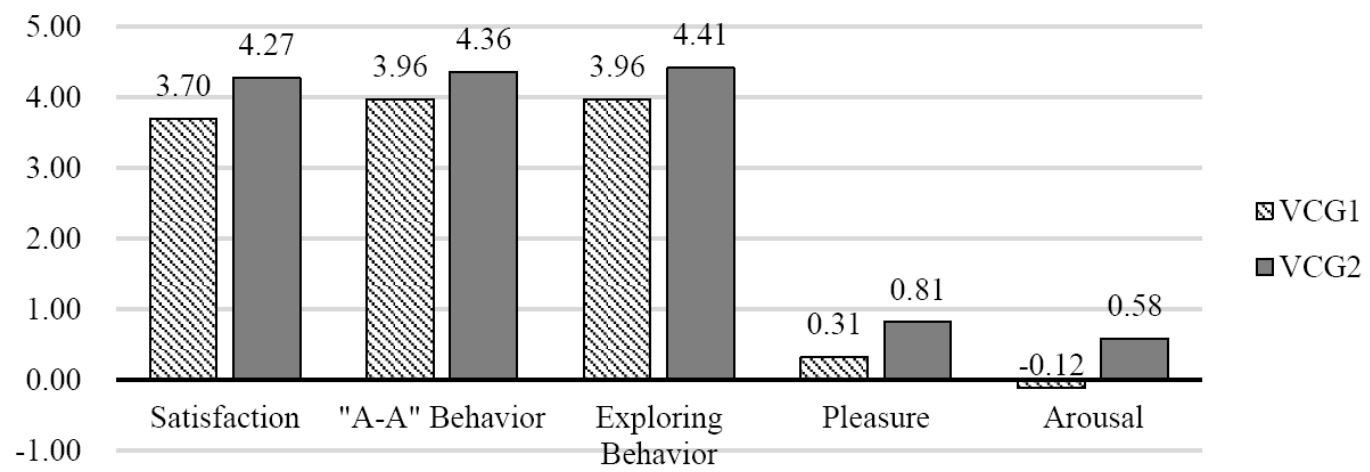

Fig. 11. Comparison of Individual Responses between the Two Green Scenes.

In the two red scenes, there was no significant difference in individual responses from the paired sample test, indicating that there is no significant difference in individual responses between the two scenes, whether it is the red sales advertisement or the red lantern. This result indicates that red mainly presents its embodied meaning in the public spaces of shopping malls, and its influence on individuals mainly depends on the color 
itself rather than on the attribute of the concrete object it carries, which further validates the results of previous studies [1,21,34].

\section{Discussion}

In the public spaces of shopping malls, the influence of hue on individual responses is very small, while the presence of a certain proportion of non-grey colors in a scene reduces satisfaction, positive behavior intention, and dominance of the environment, which further verifies the conclusion obtained in previous studies [3, 11]. Yet, the scenes in which red (a color with a longer wavelength) is added enhance the degree of arousal $[3,11]$. In previous studies, the colors were usually set at a high saturation to compare the influence of warm and cool colors on individuals. It can be concluded that warm colors make people want to be close, while cool colors have the opposite effect [1]. The field investigation showed that in the real store environment, the overall color tends to be grey, while the warm and cool colors with high saturation only account for a small proportion. In this study, the setting of colors is close to the actual environment. Therefore, compared with the theoretical meaning of previous studies, this study has more practical meaning.

Overall, in the common mall environment (where color saturation is usually less than $30 \%$ ), the lower the average saturation, that is, the greyer the overall color of the whole picture, the higher the satisfaction level of the participants, the more "approach"-leaning the behavior, and the higher the degree of pleasure and dominance in the emotion responses. Comparing the effect of $\mathrm{S}, \mathrm{S}_{\mathrm{MC} 1}$, and $\mathrm{S}_{\mathrm{CC} 3}$ on the individual responses, it is observed that the overall impact trend is similar, but $R^{2}$ of $S_{M C 1}$ and $S_{C C 3}$ is higher, as one of the largest proportions of pixels in the scene of a primary color and the distribution of the most popular $\mathrm{CC} 3$ of the saturation effect on the individual responses are more regular.

Compared with previous studies, Guilford and Smith [2] found that colors with a high saturation make people happy, whereas Schloss et al. [5] specifically discussed the influence of colors at different saturation levels on evaluation. Although people reported higher preference evaluation scores when they were faced with pure color squares with a high saturation, they preferred specific items with a low saturation. In the present study, the above conclusion is further advanced. In the process of colors progressing from color squares to specific objects, and then to the constituent environment, the lower the saturation, the better the evaluation and the more "approach"-leaning the behavior. In the study of Schloss et al. [5], the color blocks and objects were the foreground scenery in the participants' visual fields, and people evaluated the colors and objects themselves. However, here, colors constitute the environment and colors with a high saturation provide too much visual information, which weakens the information of the task to be processed by the participants. The participants are distracted, thereby reducing the degree of control over the environment and causing a series of evaluation degradations.

By analyzing the relationship between brightness and individual responses, it is observed that the brightness difference between several main colors in a scene is more important than the influence of the brightness value itself. Additionally, there is no significant correlation between $\Delta \mathrm{B}_{13}$, the brightness difference of the two colors of the proportion of pixels, which varies significantly, and individual responses. This indicates that the colors 
contributing to the brightness difference need to occupy pixels of similar scale to affect individual responses significantly. Simultaneously, a color with a smaller percentage of pixels may have a very high or very low brightness; although they can contribute to a large brightness difference, there is no significant influence on individual responses.

It should be noted that, to ensure the rationality of the existence of the target color in the scenes, the two pairs of pictures used to study the embodied and referential meanings of color also carry the meaning information of specific items. In one of the images of each pair, there are advertising boards and in the other, there is a different item. Among them, lanterns are red, which may make people think of a "festival" or "wedding" in the Chinese culture. However, there is no significant difference of the two scenes. This indicates that red color influences people by its embodied meaning rather than the referential meaning. Plants were green, which people may associate with "natural" and "fresh". Follow-up studies should be conducted to focus on whether the same results are obtained for the same items with different colors as well as whether the meaning information of items can be blurred by image processing.

When designing and managing the shopping mall environment, the overall color should be set to greyish, forming a relatively unified visual background, which can improve the overall satisfaction of the public space, and, thus, achieve an improvement in the evaluation of the overall shopping mall. If a high saturation color needs to be increased due to special sales, festivals, and other activities, it is suggested to control the proportion of the color and take an appropriately warm color. At the same time, in areas that need to attract the consumers' attention, the combination of visual elements with similar areas and large differences in brightness can be designed to create a certain visual effect of contrast.

\section{Conclusions}

Aiming at exploring the influence of environment color on individual responses in the public spaces of shopping malls, a field survey and a laboratory experiment were conducted. From these, the following conclusions were drawn:

In the real public spaces of shopping malls, the hues of colors are mainly red, orange, and yellow, and some scenes are supplemented by blue. Moreover, the saturations of over $90 \%$ of colors are lower than $33 \%$, and the range of brightness is very wide and evenly distributed.

Hue has a slight effect on individual responses, wherein scenes with red evoke people's arousal, and other individual responses decrease when there is blue or red in a scene. Saturations of a whole scene, of the main color of a scene, and of the most widely distributed color, can influence people's satisfaction, behavior intention, and emotion responses (except arousal) significantly. Lower saturation obtains higher satisfaction and approach behavior, especially for actions. Moreover, the brightness difference between two colors with a similar pixel proportion in a scene can affect individual responses significantly.

Furthermore, the effect of color on individual responses is also related to the carrier of the color. Red color is more associated with embodied meaning regardless of the specific object which it occupies, whereas green color is more associated with referential 
Fashu Yi and Jian Kang: Color Research and Application doi: $10.1002 / \mathrm{col} .22478$

meaning, in which different objects have significantly different influences. Considering the different effects of colors can more accurately adjust a person's feeling with regard to a certain aspect. 


\section{References}

[1] Labrecque LI, Patrick VM, Milne GR. The Marketers' Prismatic Palette: A Review of Color Research and Future Directions. Psychol Market. 2013;30(30):187-202.

[2] Guilford JP, Smith PC. A system of color-preferences. Am J Psychol. 1959;72(4):487.

[3] Crowley AE. The two-dimensional impact of color on shopping. Market Lett. 1993;4(1):59-69.

[4] Ou LC, Luo MR, Woodcock A, Wright A. A study of colour emotion and colour preference. Part III: Colour preference modeling. Color Research \& Application. 2004;29(5):381-389.

[5] Schloss KB, Strauss ED, Palmer SE. Object Color Preferences. Color Research \& Application. 2013;38(6):393-411.

[6] Elliot AJ, Maier MA. Color and Psychological Functioning. Curr Dir Psychol Sci. 2007;16:250-254.

[7] Elliot AJ, Maier MA. Color-in-Context Theory[M]. Advances in Experimental Social Psychology, Burlington: Acodemic Press, 2012, 61-125.

[8] Valdez P, Mehrabian A. Effects of color on emotions. Journal of Experimental Psychology General. 1994;123(123):394-409.

[9] Valberg A. Light Vision Color[M]. Chichester: John Wiley \&Sons, Ltd, 2005.

[10] Lakens D, Fockenberg DA, Lemmens KPH, Ham J, Midden CJH. Brightness differences influence the evaluation of affective pictures. Cognition Emotion. 2013;27(7):1225-1246.

[11] Mehta R, Zhu RJ. Blue or Red? Exploring the Effect of Color on Cognitive Task Performances. Science. 2009;323(5918):1226-1229.

[12] Kotler P. Atmospherics as a Marketing Tool. J Retail. 1973;49(4):48-64.

[13] Bellizzi JA, Hite RE. Environmental color, consumer feelings, and purchase likelihood. Psychol Market. 1992;9(5):347-363.

[14] Babin BJ, Hardesty DM, Suter TA. Color and shopping intentions: The intervening effect of price fairness and perceived affect. J Bus Res. 2003;56(7):541-551.

[15] Bellizzi JA, Crowley AE, Hasty RW. The Effects of Color in Store Design. J Retailing. 1983;59(1):21-45.

[16] Terwogt MM, Hoeksma JB. Colors and Emotions: Preferences and Combinations. J Gen Psychol. 1995;122(1):5.

[17] Chebat J, Morrin M. Colors and cultures: Exploring the effects of mall décor on consumer perceptions. J Bus Res. 2007;60(3):189-196.

[18] Hurlbert AC, Ling Y. Biological components of sex differences in color preference. Current Biology Cb. 2007;17(16):R623-R625.

[19] Palmer SE, Schloss KB. Ecological Valence and Human Color Preferences. Color and Imaging Conference, 18th Color and Imaging Conference Final Program and Proceedings. 2011: 145-153.

[20] Palmer SE, Schloss KB. An ecological valence theory of humancolor preference. Proc Natl Acad Sci USA. 2010;107(19):8877.

[21] Zhu R, Meyerslevy J. Distinguishing between the meanings of music: when background music affects product perceptions. J Mark Res. 2005;42(3):333-345.

[22] Pheasant RJ, Fisher MN, Watts GR, Whitaker DJ, Horoshenkov KV. The importance of auditory-visual interaction in the construction of "tranquil space". J Environ Psychol. 2010;30(4):501-509. 
[23] Zhao W. Optimization design of acoustic and daylight environment for commercial building atria. 2016; $\mathrm{PhD}: 242$.

[24] Yi F, Kang J, Jin H. Research on space matching of large shopping malls based on modern commercial functional requirements. The 15th China Architecture Science and Technology Symposium, Harbin, China 2014:100-105.

[25] Pan Y, Meng Z. Influence of attention in visual-auditory on visual masking effect. Video Engineering. 2011;9:109-111,128.

[26] Osgood CE, Suci GJ, Tannenbaum PH. The Measurement of Meaning, University of Illinois Press, 1957:342.

[27] Rosenberg MJ. Attitude organization and change: an analysis of consistency among attitude components. Yale University Press, 1960.

[28] Mehrabian A, Russell JA. An approach to environmental psychology. [M]. Cambridge, MA: The MIT Press, 1974.

[29] Mehrabian A. Pleasure-arousal-dominance: a general framework for describing and measuring individual differences in temperament. Curr Psychol. 1996;14(4):261-292.

[30] Donovan R, Rossiter JR. Store Atmosphere: an environment psychology approach. J Retail. 1982;58:34-57.

[31] Floyd MF. Pleasure, arousal, and dominance: exploring affective determinants of recreation satisfaction. Leis Sci. 1997;19(2):83-96.

[32] Pheasant R, Horoshenkov K, Watts G, Barrett B. The acoustic and visual factors influencing the construction of tranquil space in urban and rural environments tranquil spaces-quiet places? J Acoust Soc Am. 2008;123(3):1446-1457.

[33] Watts GR, Pheasant RJ. Tranquillity in the Scottish highlands and Dartmoor National Park-the importance of soundscapes and emotional factors. Appl Acoust. 2015;89:297-305.

[34] Meyers-Levy J, Zhu RJ. Gender differences in the meanings consumers infer from music and other aesthetic stimuli. J Consum Psychol. 2010;20(4):495-507.

[35] Ba, M., \& Kang, J. (2019). A laboratory study of the sound-odour interaction in urban environments. Building and Environment, 147, 314-326. https://doi.org/10.1016/j.buildenv.2018.10.019.

[36] Ren, X., \& Kang, J. (2015). Effects of the visual landscape factors of an ecological waterscape on acoustic comfort. Applied Acoustics, 96, 171-179. https://doi.org/10.1016/j.apacoust.2015.03.007. 